\title{
The Vaccine and Cervical Cancer Screen project 2 (VACCS 2): Linking cervical cancer screening to a two-dose HPV vaccination schedule in the South- West District of Tshwane, Gauteng, South Africa
}

\author{
L C Snyman, ${ }^{1}$ MB ChB, MPraxMed, MMed (O\&G), FCOG (SA); G Dreyer, ${ }^{1}$ MB ChB, MMed (O\&G), MCOG (SA), PhD; \\ C Visser, ${ }^{1}$ BSc (Hons), MSc; M H Botha, ${ }^{2}$ MB ChB, MMed (O\&G), FCOG (SA), PhD; F H van der Merwe, ${ }^{2}$ MB ChB, MMed (O\&G), FCOG \\ ${ }^{1}$ Gynaecological Oncology Unit, Faculty of Health Sciences, Department of Obstetrics and Gynaecology, University of Pretoria, South Africa \\ ${ }^{2}$ Unit for Gynaecological Oncology, Department of Obstetrics and Gynaecology, Faculty of Medicine and Health Sciences, Stellenbosch University, \\ Tygerberg, Cape Town, South Africa
}

Corresponding author: L C Snyman (leon.snyman@up.ac.za)

\begin{abstract}
Background. Cervical cancer is a preventable disease with a high prevalence in South Africa (SA), where screening is opportunistic. Primary prevention is now possible through HPV vaccination. In VACCS 1 the feasibility of linking cervical cancer with HPV vaccination was demonstrated.

Objectives. To investigate the feasibility of linking HPV self-testing with a two-dose HPV vaccination schedule and to compare results with VACCS 1.

Methods. The project was conducted in five schools in the South-West District of Tshwane, Gauteng, SA. Leaflet information on cervical cancer and screening was provided, with requests for consent and assent for a two-dose HPV vaccination of schoolgirls. Female caregivers were invited to take part in HPV self-screening.

Results. Of 965 girls invited for vaccination, 519 (53.7\%) had full consent and 518 (99.8\%) received at least one vaccine dose. The invited uptake rate was $53.7 \%$ and 495 girls received both doses, giving a completion rate of $95.4 \%$ v. $82.6 \%$ in VACCS 1 . Of 1135 self-screen kits handed out, 560 (49.3\%) were not returned. The mean age (standard deviation) of the 160 women who participated in self-screening was 38.7 (7.7) years. HPV testing was negative in 116 women (72.5\%), 15 women (9.4\%) tested positive for HPV 16 and/or 18, and 27 (16.9\%) were positive for non-16/18 oncogenic HPV.

Conclusion. Data from the VACCS projects suggest that school-based vaccine programmes can be successfully implemented. A two-dose schedule allowed for higher completion rates. Linking self-collected HPV screening to HPV vaccination is feasible, is a promising and viable screening strategy, and reached the appropriate age group for screening.
\end{abstract}

S Afr Med J 2015;105(3):191-194. DOI:10.7196/SAMJ.8888

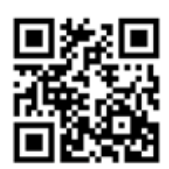

Cervical cancer incidence rates in South Africa (SA) have reached epidemic proportions, where cervical cancer accounts for 18.0/100 000 of deaths (age-standardised rate). Cancer of the cervix is the leading cause of cancer-related death in Africa. ${ }^{[1]}$ HIV infection increases the risk of developing cervical cancer and lowers the age at diagnosis. ${ }^{[2]}$

Although the disease is largely preventable through screening programmes aimed at treatment of detected cancer precursor lesions, successful screening programmes have not been implemented anywhere in the developing world. ${ }^{[3]}$ Fortunately effective vaccines are registered and available against the viral cause of the disease, namely human papillomavirus (HPV) ${ }^{[4]}$ Before 2014, these vaccines were not yet introduced into the national vaccine programme in SA - the Expanded Program on Immunization (EPI). During 2014, a two-dose vaccination programme was introduced in some schools for girls in grade 4 in SA. The vaccines are available for private healthcare users, but are underutilised in this sector as well. It is predicted that in the future cervical cancer vaccination will be a more cost-effective and much more effective strategy than screening for this disease. ${ }^{[5]}$

HPV vaccines were originally tested in a three-dose regimen in adult women and found to be highly immunogenic and effective in preventing cervical cancer precursor lesions. Recently new data have demonstrated that vaccination with two doses of the same vaccine in younger girls (aged 9 - 14 years) also provides high levels of antibodies. Indeed, the antibody response was demonstrated to be non-inferior to the immunogenicity obtained with a two-dose schedule in females aged $15-25$ years. A similar safety profile was also demonstrated when compared with the three-dose group. ${ }^{[6,7]}$

Countries in the developed and developing world are increasingly opting to provide two doses of HPV vaccine as part of their national policy in response to these preliminary data. This method of bridging to efficacy data is a well-established approach in vaccinology. It is proposed that introduction of the HPV vaccine into the EPI programme in SA will be greatly facilitated by providing only two doses (at months 0 and 6) to primary school girls, while immunogenicity will be uncompromised because of the young age of the recipients. ${ }^{[7]}$ Herd immunity will depend upon high uptake and completion rates, while affordability and success of the programme will also depend upon resources needed as well as direct and indirect programme costs.

The first arm of the Vaccine and Cervical Cancer Screen (VACCS) project (VACCS 1) showed that cervical cancer screening of women with self-testing can be successfully linked to implementation of an HPV vaccination programme at the schools attended by their daughters. In this trial, the conventional three-dose regimen was used and more than 1000 girls were vaccinated. ${ }^{[8]}$ 
The current study, done in the same district as VACCS 1, aimed to investigate the feasibility of linking two-dose vaccination of eligible primary school girls with HPV self-testing of their female caregivers. In addition, this study compared some differences in methodology and outcomes with the first VACCS project. Screening uptake and outcomes were also investigated and compared with the outcomes achieved in VACCS 1 . The major differences in study methods between VACCS 1 and 2 included the invitation to female parents and guardians to take part in self-screening, the two-dose vaccination schedule, and the collection and testing of HPV DNA. HPV tests were sent with the girls attending school and, unlike with VACCS 1, there were no additional information events in the form of an oral presentation presented by a doctor.

\section{Methods}

As with the first VACCS project, this national study was conducted in Gauteng and Western Cape provinces with the approval of the national and provincial departments of Basic Education and Health. The method and results of the Gauteng part of the study are described here.

Five primary schools neighbouring those in which girls had been vaccinated in VACCS 1 were identified in the South-West District of Tshwane. After obtaining consent from the governing body and principal of each school, study packs containing printed leaflet information, informed consent and assent forms and self-screen kits were handed out to all the girls in grades 4 - 7 (aged $\geq 9$ years) to take home.

The printed leaflet contained information about cervical cancer and its symptoms, accurate and complete information about the safety and efficacy of the HPV vaccine for primary prevention of cervical cancer, and information on screening for the disease. Parents and guardians of eligible girls were requested to provide informed consent for a two-dose HPV vaccine schedule, while all eligible girls were requested to provide assent for vaccination. Two bivalent vaccine doses donated by the manufacturing company were administered to the consented girls, 6 months apart, by a team of registered nurses during school hours.

Female parents and guardians of eligible girls were invited to take part in self-administered HPV screening. The screen kit consisted of a sample collector with user instructions available as an Evalyn brush. Specimens together with personal information were returned to the school in sealed containers. DNA was extracted from the Evalyn brush and tested using Roche Cobas 4800. ${ }^{[9]}$ HPV DNA results were reported as positive for HPV 16, HPV 18 and other high-risk HPV (hrHPV) types $(31,33,35,39,45,51,52,56,58,59,66,68)$ or negative for oncogenic HPV.

HPV test results were interpreted as positive if DNA of any of the 15 high-risk viral types was found, and were interpreted as invalid if no DNA amplification occurred as tested by the internal control.

Women who screened positive were informed of the results via the school system and by making use of mobile phone technology, and invited to the gynaecology department at Kalafong Hospital for treatment or requested to visit their own healthcare provider with the screen results.

\section{Definitions}

The invited cohort (IC) was defined as all female learners enrolled in the selected schools in grades $4-7$ and the consented cohort (CC) as participants with written consent from the parent or guardian as well as assent from the learner. Girls whose parents or guardians had given consent but who did not attend vaccine events were included in the CC. The vaccinated cohort (VC) comprised all girls who received at least one vaccine dose. Vaccine uptake rates were calculated in a number of ways in order to allow comparison with other published HPV vaccine reports and with VACCS 1 . The consented uptake rate (CUR) was calculated as VC/CC and the invited uptake rate (IUR) as VC/IC.

Vaccine completion rates (VCRs) were determined using the vaccine cohort as a denominator. The VCR was calculated using all girls who received both vaccine doses, while the insufficiently vaccinated rate (IVR) used the number of girls who received only one vaccine dose.

The study was approved by the Research Ethics Review Committee of the Faculty of Health Sciences, University of Pretoria (90/2013).

\section{Results}

\section{Vaccination data}

In the five schools selected for the project, the IC consisted of 965 girls. The CC consisted of 519 girls (53.7\%) of whom 518 received the first vaccine dose (VC), giving a consented uptake rate of $99.8 \%$. The IUR was $53.7 \%$. Four hundred and ninety-five girls received both doses and 23 received only one dose, resulting in an IVR of $4.4 \%$. The VCR was $95.4 \%$ for two doses.

The vaccination uptake and completion rates for the five different schools are shown in Table 1. One adverse event was reported as a rash developing a few hours after vaccination in a girl known to be allergic to various substances, but could not be confirmed to be related to the vaccine.

\section{Screening}

A total of 1135 self-screen kits were handed out to eligible girls attending the five schools, to be passed on to their female parents and guardians. Female staff members at the schools also requested selfscreen kits, which accounts for the difference between the numbers of tests handed out and the IC. Of the 1135 self-screen kits handed out, 378 (33.3\%) were returned unused and undamaged, 37 (3.3\%) were unused but damaged, and $560(49.3 \%)$ were not returned. Unused but damaged kits were not tested for the presence of HPV DNA.

One hundred and sixty women participated in self-screening. The return rate as a proportion of tests distributed was $14.1 \%$. Screening uptake was $16.6 \%$ when calculated as a proportion of the IC (160 from $965)$ and $30.8 \%$ when calculated as a proportion of the CC (160 from 519). The proportion of the $C C$ was calculated to compare with similar

Table 1. Vaccination uptake and completion rates for the different schools

\begin{tabular}{|c|c|c|c|c|c|c|c|c|c|}
\hline School & IC, $n$ & CC, $n$ & VC, $n$ & IUR, \% & CUR, \% & Received 2 doses, $n$ & VCR, \% & Received 1 dose, $n$ & IVR, \% \\
\hline 1 & 351 & 156 & 156 & 44.4 & 100.0 & 149 & 95.5 & 7 & 4.5 \\
\hline 2 & 203 & 136 & 135 & 66.5 & 99.3 & 128 & 94.1 & 7 & 5.2 \\
\hline 3 & 226 & 120 & 120 & 53.1 & 100.0 & 113 & 94.2 & 7 & 5.8 \\
\hline 4 & 25 & 16 & 16 & 64.0 & 100.0 & 16 & 100.0 & 0 & 0.0 \\
\hline 5 & 160 & 91 & 91 & 56.8 & 100.0 & 89 & 97.8 & 2 & 2.2 \\
\hline Total & 965 & 519 & 518 & 53.7 & 99.8 & 495 & 95.4 & 23 & 4.4 \\
\hline
\end{tabular}


uptake rates calculated in VACCS $1 .^{[8]} \mathrm{A}$ comparison of the different screen uptake rates is presented in Table 2.

When calculating screening uptake, the size of the true target population needs to be clarified. Although not investigated in the current study, data from the questionnaires administered to a similar population during the VACCS 1 study suggested that at least $52.6 \%$ of women were in need of a screening test ('had last test $>10$ years ago'). Using these data, the true target population in VACCS 2 is 538 women, corresponding to an uptake of $29.7 \%$.

The mean (standard deviation) age of women participating in self-screening was 38.7 (7.7) years (range 20 - 67). The age distribution for this population is shown in Fig. 1.

Of the 160 women tested, 2 (1.3\%) had invalid tests and $116(72.5 \%)$ tested negative for hrHPV. Fifteen women $(9.4 \%)$ tested positive for HPV 16 and/or 18 and 27 (16.9\%) tested positive for non-16/18 hrHPV (Fig. 2). Of the 15 women who tested positive for HPV 16/18, 3 had only HPV 16 and 1 had only HPV 18 . Eleven women tested positive for both HPV 16 and 18 . These screening results per age group are shown in Fig. 2. The comparative data from VACCS 1 are shown in Table 3.

\section{Discussion}

Reported vaccine uptake differs widely and is influenced by many factors such as social, religious, cultural and awareness aspects. ${ }^{[10]}$ School-based vaccination programmes also tend to be more effective than other programmes. ${ }^{[11]}$ In this study, informed consent from parents as well as assent from the girls were prerequisites for inclusion in the vaccine cohort. A vaccine uptake rate of $53.7 \%$, calculated as a proportion of all girls eligible for vaccination, compares well with reports from other parts of the world. It is lower than the $64.0 \%$ overall uptake reported for VACCS $1^{[12]}$ and can be attributed to the modification in information transfer. In VACCS 1 the vaccine uptake was $51.7 \%$ for girls whose parents or guardians did not attend the information sessions and $87.5 \%$ for those whose parents or guardians attended. Although such information events result in higher uptake rates, they are not sustainable during a mass vaccine roll-out programme. Higher vaccine uptake rates can be accomplished by introducing an opt-out programme combined with assent from girls.

Several studies have confirmed the noninferiority of two vaccine doses compared with three doses. ${ }^{[6,7]}$ The VCR of $95.4 \%$ achieved during this study compares favourably with other published data from $\mathrm{SA}^{[13]}$ and with the sufficiently vaccinated rate (two doses 6 months apart) of $94.5 \%$ in Gauteng schools vaccinated in one calendar

year during VACCS $1 .{ }^{[8]}$ A vaccine programme in which two doses are administered should

\section{Table 2. Comparison of different screen uptake rates for VACCS 1 and VACCS 2}

\begin{tabular}{lll}
\hline Participation rates & VACCS 1, \% & VACCS 2, \% \\
\hline Return rate: Self-test kits returned used/self-test kits distributed & 31.8 & 14.1 \\
Participation rate: IC & 15.3 & 16.6 \\
Participation rate: CC & 23.9 & 30.8
\end{tabular}

Table 3. Comparison of HPV screen outcomes for VACCS 1 and 2

\begin{tabular}{lll}
\hline HPV self-screen & $\begin{array}{l}\text { VACCS 1 (Roche Linear } \\
\text { Array and tampons), \% }\end{array}$ & $\begin{array}{l}\text { VACCS 2 (Cobas and } \\
\text { Evalyn samplers), \% }\end{array}$ \\
\hline Invalid tests & 3.6 & 1.3 \\
hrHPV-negative & 66.7 & 72.5 \\
Any hrHPV & 29.6 & 26.3 \\
HPV 16- and/or 18-positive & 9.1 & 9.4 \\
Non-16/18 hrHPV-positive & 20.6 & 16.9
\end{tabular}

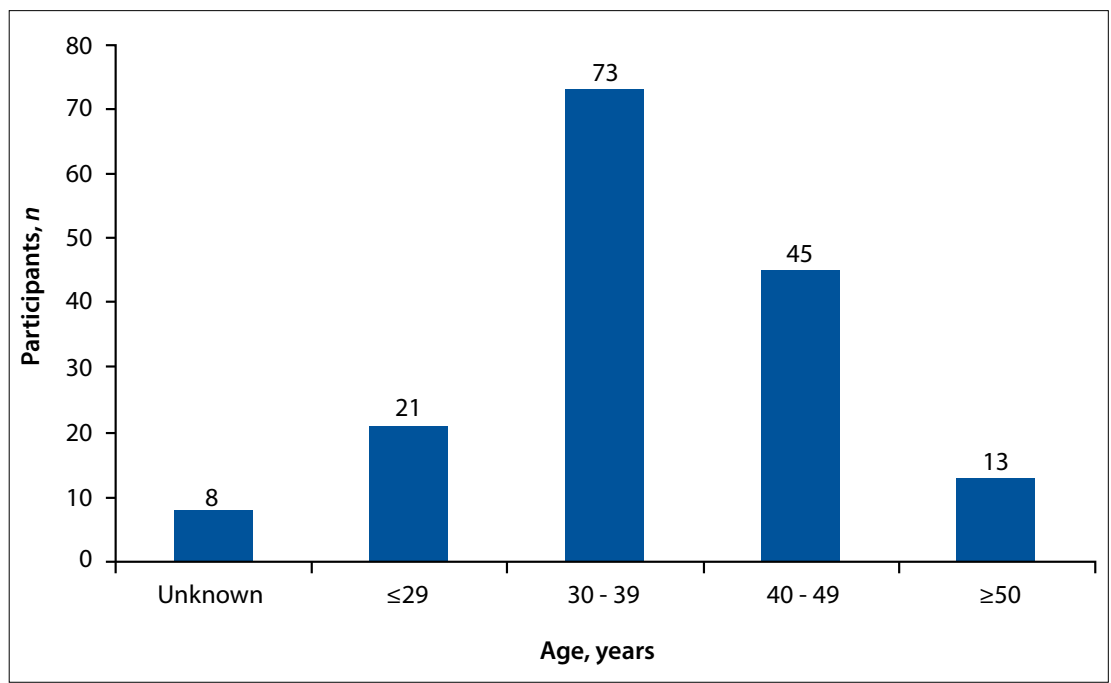

Fig. 1. Age distribution of women participating in self-screening.

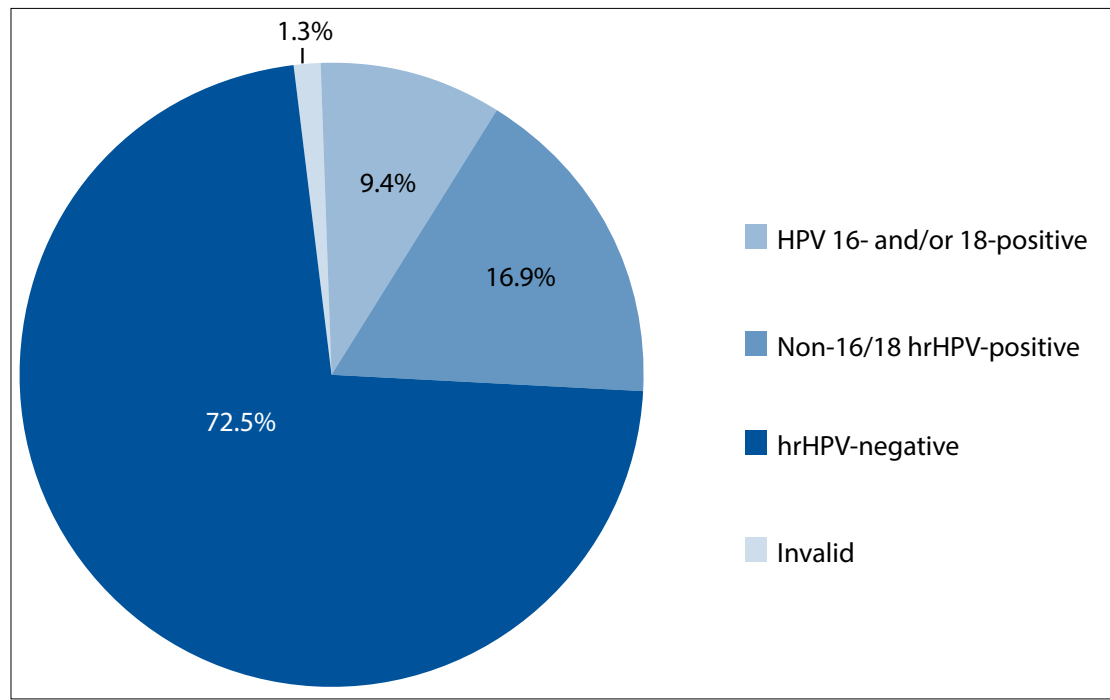

Fig. 2. HPV self-testing screen results. 


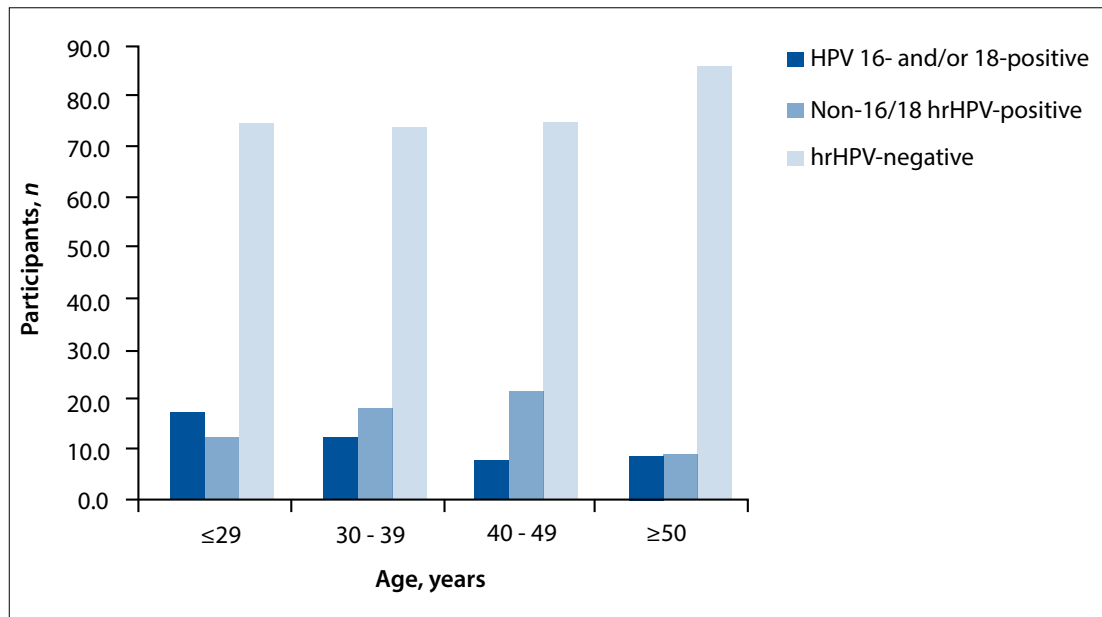

Fig. 3. Age distribution of participants.

be less complicated to implement and more cost-effective, and results in the vaccination of more individuals. ${ }^{[7]}$

Screening for cervical cancer using conventional cytology has had limited success in $\mathrm{SA}^{[1]}$ despite being effective and having been available for more than 50 years. It is therefore vital to investigate alternative screening options and screening opportunities. Selftesting for HPV as a screening tool for cervical cancer has been well documented and can be implemented even in resource-poor settings. [14-16] Both VACCS 1 and 2 investigated the feasibility of implementing HPV self-screening in an urban population without making use of the health system. The method followed in the current study of inviting female parents and guardians to participate in screening and to distribute information about the vaccination of their children in an information package delivered at home via the children is a realistic, feasible and cost-effective alternative compared with a strategy that has not been widely implemented. The screened population falls largely into the ideal age group of women requiring screening (Fig. 1).

In this study, screening uptakes compared favourably with similar calculations from VACCS 1 despite the different methods of providing information. In VACCS 1 , self-test kits were distributed to women after they had attended lectures at information events, while in VACCS 2 self-test kits were distributed to all female parents and guardians together with written information about cervical cancer, screening and vaccination. In comparable studies conducted on European non-responders via a mailed invitation, compliance rates varied between $6.4 \%$ (UK) and $31.3 \%$ (Netherlands), with most groups reporting uptake rates around $30 \%{ }^{[17]}$ Screening uptakes achieved during this study of $30.8 \%$ (CC) and $29.7 \%$ (unscreened cohort) compare favourably with these data.
When comparing the results, it is important to note that the screening methodology used in VACCS 1 and VACCS 2 differed in two important aspects: (i) VACCS 1 employed tampon-based self-screening transported in a buffer solution, while Evalyn brushes were used for self-collection and transported dry in VACCS 2; and (ii) different HPV testing technology was used: Roche linear array in VACCS 1 , and Roche Cobas 4800 in VACCS 2.

\section{Conclusion}

Data from the VACCS project suggest that school-based vaccine programmes can be implemented successfully in suburban areas. A two-dose vaccine schedule yields higher VCRs and will also allow for more girls to be vaccinated. Results from the current study support the SA Department of Health HPV vaccination programme.

Screening uptake and results in this study confirm the feasibility of HPV screening in this population. Linking self-testing HPV screening to $\mathrm{HPV}$ vaccination is a promising alternative to the current screening policy. The screening method used in this study was successful in reaching the appropriate target population, uptake rates were acceptable, and results were effectively communicated via the school system and mobile phone technology. Screening options other than conventional population-based cervical cytology are promising and should be further investigated and implemented.

Acknowledgements. The assistance of the following groups and persons that enabled the successful completion of this project is gratefully acknowledged. Financial support was received from the Cancer Research Initiative of South Africa, a national collaborative research programme supported by the South African Medical Research Council and the Cancer Association of South Africa, and from First for Women Insurance for screening and treatment of screen-positive women and investigator support. Funding for this study was provided by GlaxoSmithKline Biologicals SA (117280). GlaxoSmithKline Biologicals SA was provided the opportunity to review a preliminary version of this manuscript for factual accuracy, but the authors are solely responsible for final content and interpretation. The authors received no financial support or other form of compensation related to the development of the manuscript. Teams of registered nurses handled vaccine processes, and Dr Karin Richter managed the laboratory screening data.

\section{References}

1. ICO (Institut Català d'Oncologia) Information Centre on HPV and Cancer. South Africa. Human papillomavirus and related cancers, fact sheet 2013. http://www.hpvcentre.net/statistics/reports/ZAF FS.pdf (accessed 17 April 2014).

2. Parkin DM, Sitas F, Chirenje M, Stein L, Abratt R, Wabinga H. Part I: Cancer in indigenous Africans - burden, distribution, and trends. I: Cancer in indigenous Africans - burden, distribution, and trends.
Lancet Oncol 2008;9(7):683-692. [http://dx.doi.org/10.1016/S14702045(08)70175-X]

3. Gakidou E, Nordhagen S, Obermeyer Z. Coverage of cervical cancer screening in 57 countries: Low average levels and large inequalities. PLoS Med 2008;5(6):e132. [http://dx.doi.org/10.1371/journal. pmed.0050132]

4. Harper DM, Franco EL, Wheeler CM, et al. Sustained efficacy up to 4.5 years of a bivalent $\mathrm{L} 1$ virus-like particle vaccine against human papillomavirus types 16 and 18: Follow-up from a randomised control trial. Lancet 2006;367(9518):1247-1255. [http://dx.doi. org/10.1016/S0140-6736(06)68439-0]

5. Villa LL, Costa RL, Petta CA, et al. High sustained efficacy of a prophylactic quadrivalent human papillomavirus types 6/11/16/18 L1 virus-like particle vaccine through 5 years of follow-up. $\mathrm{Br}$ J Cancer 2006;95(11):1459-1466. [http://dx.doi.org/10.1038/ s.bjc.6603469]

6. Dobson SR, McNeil S, Dionne M, et al. Immunogenicity of 2 doses of HPV vaccine in younger adolescents vs 3 doses in young women: A randomized clinical trial. JAMA 2013;309(17):1793-802. [http:// dx.doi.org/10.1001/jama.2013.1625]

7. Romanowski B, Schwarz TF, Ferguson LM, et al. Immune response to the HPV-16/18 AS04-adjuvanted vaccine administered as a 2-dose or 3-dose schedule up to 4 years after vaccination: Results from a randomized study. Hum Vaccine Immunother 2014;10(5):1155-1165. [http://dx.doi.org/10.4161/hv.28022]

8. Snyman LC, Dreyer G, Botha MH, van der Merwe, FH, Becker PJ. The Vaccine and Cervical Cancer Screen (VACCS) project: Linking cervical cancer screening to HPV vaccination in the SouthWest District of Tshwane, Gauteng, South Africa. S Afr Med J 2015;105(2):115-120. [http://dx.doi.org/10.7196/SAMJ.8418]

9. Heideman DA, Hesselink AT, Berkhof J, et al. Clinical validation

9. Heideman DA, Hesselink AT, Berkhof J, et al. Clinical validation
of the cobas $4800 \mathrm{HPV}$ test for cervical screening purposes. J Clin of the cobas $4800 \mathrm{HPV}$ test for cervical screening purposes. J Clin
$\begin{array}{ll}\text { Microbiol } & \text { 2011;49(11):3983-3985. } \\ \text { [http://dx.doi.org/10.1128/ }\end{array}$ Microbiol 2011;

10. Brewer NT, Fazekas KI. Predictors of HPV vaccine acceptability: A theory-informed, systematic review. Prev Med 2007;45(2-3):107114. [http://dx.doi.org/10.1016/j.ypmed.2007.05.013]

11. Brotherton JM, Murray SL, Hall MA, et al. Human papillomavirus vaccine coverage among female Australian adolescents: Success of the school-based approach. Med J Aust 2013;199(9):614-617. [http:// dx.doi.org/10.5694/mja13.10272]

12. Botha MH, van der Merwe FH, Snyman LS, Dreyer G. The Vaccine and Cervical Cancer Screen (VACCS) project: Acceptance of human papillomavirus vaccination in a school-based programme in two provinces of South Africa. S Afr Med J 2015;105(1):40-43. [http:// provinces of South Africa. S Afr

13. Moodley I, Mubaiwa V, Tathiah N, Denny L. High uptake of Gardasil vaccine among $9-12$-year-old schoolgirls participating Gardasil vaccine among 9 - 12-year-old schoogigirls participating
in an HPV vaccination demonstration project in KwaZuluNatal Province. S Afr Med J 2013;103(5):318-321. [http://dx.doi. org/10.7196/SAMJ.6414]

14. Mnisi EF, Dreyer G, Richter KL, Horton A, Snyman LC. Human papillomavirus DNA testing on self-collected vaginal tampon samples as a cervical cancer screening test in a Gauteng population. S Afr J Gynaecol Oncol 2013;5(2):S15-S20.

15. Richter KL. Understanding and incorporating human papillomavirus testing in cervical cancer screening: A South African perspective. S Afr J Gynaecol Oncol 2011;3(1):9-14.

16. Cuzick J, Arbyn M, Sankaranarayanan R, et al. Overview of human papillomavirus-based and other novel options for cervical cancer screening in developed and developing countries. Vaccine 2008;26(Suppl 10):K29-K41. [http://dx.doi.org/10.1016/j.
vaccine.2008.06.019] vaccine.2008.06.019]

77. Racey CS, Withrow DR, Gesink D. Self-collected HPV testing improves participation in cervical cancer screening: A systematic review and meta-analysis. Can J Pub Health 2013;104(2):el59-el66.
[http://www.ncbi.nlm.nih.gov/pubmed/23618210]

Accepted 12 January 2015. 